\title{
Numerical validation of a consistent axisymmetric lattice Boltzmann model
}

\author{
T. Reis and T. N. Phillips \\ School of Mathematics, Cardiff University, Cardiff, CF24 4AG, United Kingdom
}

(Received 14 June 2007; published 11 February 2008)

\begin{abstract}
A recently derived axisymmetric lattice Boltzmann model is evaluated numerically. The model incorporates a spatially and temporally varying source term into the evolution equation for the momentum distribution function on a two-dimensional Cartesian lattice. The precise form of the source term is derived through a Chapman-Enskog analysis so that the additional axisymmetric contributions in the Navier-Stokes equations are furnished when written in the cylindrical polar coordinate system. The validity of the model is confirmed by simulating Hagen-Poiseuille flow. Numerical predictions for the drag coefficient in Stokes' flow over a sphere are presented and shown to be in excellent agreement with analytical results. At larger Reynolds numbers the numerical predictions are shown to approach an empirically derived formula for the drag coefficient.
\end{abstract}

DOI: 10.1103/PhysRevE.77.026703

PACS number(s): 47.11.- j

\section{INTRODUCTION}

The formulation of the standard lattice Boltzmann model for predicting the flow of incompressible fluids is based on the Cartesian coordinate system. However, numerous important flow problems exist for which there is axial symmetry, e.g., flow past a sphere. The computational demand required for three-dimensional lattice Boltzmann models (LBM) is considerably greater than for the two-dimensional (2D) case. Therefore an axisymmetric LBM, which will only depend on two coordinates, is highly desirable since it makes sense computationally to take advantage of any reduction in dimension that can be accrued from geometrical considerations. Note that a reduction in dimensionality also allows for greater spatial refinement through the availability of additional degrees of freedom that would have been required for the third dimension.

In a recent article [1], the authors developed a modified lattice Boltzmann equation for axisymmetric flows by following the general philosophy embodied in the paper of Halliday et al. [2] but departing from it in the way that the second-order contribution to the source term is chosen in the recovery of the lattice momentum equation. Halliday et al. [2] demonstrated how the evolution equation for the momentum distribution function within a 2D Cartesian framework may be adjusted by adding suitable source terms in order to recover the axisymmetric Navier-Stokes equations in the macroscopic limit. The first- and second-order terms in an expansion of the source term are chosen so that the terms in the lattice continuity and momentum equations, respectively, arising from the cylindrical polar coordinate system are recovered. In the article of Reis and Phillips [1], the first- and second-order contributions to the source term are derived through a Chapman-Enskog analysis. The analysis is performed consistently within the Chapman-Enskog formalism, unlike previously adopted approaches. Furthermore, the form of the source terms that are derived here allows for a more efficient implementation of the LBM for axisymmetric flow problems due to the reduction in the number of terms that require numerical differentiation.

The aim of the present paper is to perform a numerical evaluation of the modified axisymmetric LBM derived in [1] using a number of benchmark problems to demonstrate the accuracy of the axisymmetric LBM calculations. The numerical predictions are shown to be in excellent agreement with available analytical and empirical results, thus confirming the validity of the proposed model.

\section{GOVERNING EQUATIONS IN AXISYMMETRIC GEOMETRIES}

Consider the flow of an incompressible, isotropic fluid through a three-dimensional pipe. Let $\mathbf{e}_{r}, \mathbf{e}_{\theta}$, and $\mathbf{e}_{z}$ be the standard orthonormal unit vectors defining a cylindrical coordinate system:

$$
\mathbf{e}_{r}=\left(\frac{x}{r}, \frac{y}{r}, 0\right), \quad \mathbf{e}_{\theta}=\left(\frac{y}{r},-\frac{x}{r}, 0\right), \quad \mathbf{e}_{z}=(0,0,1),
$$

where $r=\sqrt{x^{2}+y^{2}}, x=r \cos \theta$ and $y=r \sin \theta$. If the solution to the Navier-Stokes equation is of the form

$$
\mathbf{u}=u_{r}(r, z) \mathbf{e}_{r}+u_{z}(r, z) \mathbf{e}_{z},
$$

that is the velocity field does not depend on $\theta$, then the flow is said to be axisymmetric (without swirl). The continuity equation in cylindrical coordinates is

$$
\frac{\partial u_{r}}{\partial r}+\frac{u_{r}}{r}+\frac{\partial u_{z}}{\partial z}=0
$$

and the components of the momentum equation are

$$
\begin{aligned}
\frac{\partial u_{r}}{\partial t}+u_{r} \frac{\partial u_{r}}{\partial r}+u_{z} \frac{\partial u_{r}}{\partial z}= & -\frac{1}{\rho} \frac{\partial P}{\partial r} \\
& +\nu\left(\frac{\partial^{2} u_{r}}{\partial r^{2}}+\frac{1}{r} \frac{\partial u_{r}}{\partial r}-\frac{u_{r}}{r^{2}}+\frac{\partial^{2} u_{r}}{\partial z^{2}}\right), \\
\frac{\partial u_{z}}{\partial t}+u_{r} \frac{\partial u_{z}}{\partial r}+u_{z} \frac{\partial u_{z}}{\partial z} & =-\frac{1}{\rho} \frac{\partial P}{\partial z}+\nu\left(\frac{\partial^{2} u_{z}}{\partial r^{2}}+\frac{1}{r} \frac{\partial u_{z}}{\partial r}+\frac{\partial^{2} u_{z}}{\partial z^{2}}\right),
\end{aligned}
$$

where $\nu$ is the kinematic viscosity.

By performing the following coordinate transformation: 


$$
\begin{gathered}
(r, z) \mapsto(y, x), \\
\left(u_{r}, u_{z}\right) \mapsto\left(u_{y}, u_{x}\right),
\end{gathered}
$$

Eqs. (3)-(5) can be written in Cartesian-type coordinates:

$$
\begin{gathered}
\partial_{\alpha} u_{\alpha}=-\frac{u_{y}}{y}, \\
\frac{D \rho u_{\alpha}}{D t}+\partial_{\alpha} P-\nu \rho \nabla^{2} u_{\alpha}=\frac{\nu \rho}{y} \partial_{y} u_{\alpha}-\frac{\nu \rho u_{\alpha}}{y^{2}} \delta_{\alpha y},
\end{gathered}
$$

where $D / D t$ is the material derivative, $\delta_{\alpha \beta}$ is the Kronecker delta function, and $\alpha=x, y$. The terms on the right-hand side of the momentum Eq. (9) are the additional axisymmetric contributions that the source terms need to recover.

\section{AXISYMMETRIC LBE}

The lattice Boltzmann equation can be classified as an explicit, Lagrangian, finite-hyperbolic approximation to the Navier-Stokes equation that has been derived within the framework of statistical mechanics. The LBE is characterized by a lattice and some rule describing the manner in which particles move along lattice directions from one node to another. A simple yet sufficiently symmetric and multispeed lattice is the D2Q9 lattice. It is a nine velocity model (including a rest particle at the center) for which an equilibrium solution with all the properties needed to recover the macroscopic hydrodynamic equations can be derived. The nine discrete velocities are given by

$\mathbf{c}_{i}=\left\{\begin{array}{cc}(0,0), & i=0, \\ (\cos [(\alpha-1) \pi / 2], \sin [(\alpha-1) \pi / 2]), & i=1, \ldots, 4, \\ \sqrt{2}(\cos [(2 \alpha-9) \pi / 4], \sin [(2 \alpha-9) \pi / 4]), & i=5, \ldots, 8,\end{array}\right.$

where we have assumed that the unit of velocity is unity. Let $N_{i}(\mathbf{x}, t)$ be the single particle distribution function at node $\mathbf{x}$ and time $t$ with momentum in the direction $\mathbf{c}_{i}$. The lattice Boltzmann evolution equation in rectilinear coordinates is

$$
\begin{gathered}
N_{i}\left(\mathbf{x}+\mathbf{c}_{i}, t+1\right)=N_{i}(\mathbf{x}, t)-\omega\left(N_{i}(\mathbf{x}, t)-N_{i}^{(e)}(\mathbf{x}, t)\right), \\
i=0, \ldots, 8,
\end{gathered}
$$

where $N_{i}^{(e)}$ is an equilibrium function and $\omega$ is the relaxation parameter of the fluid. The particular form of the equilibrium function for the D2Q9 lattice is due to Qian et al. [3]

$$
N_{i}^{(e)}=\rho w_{i}\left[1+3 \mathbf{c}_{i} \cdot \mathbf{u}-\frac{3}{2} u^{2}+\frac{9}{2}\left(\mathbf{c}_{i} \cdot \mathbf{u}\right)^{2}\right],
$$

where $\quad w_{0}=4 / 9, \quad w_{i}=1 / 9 \quad(i=1, \ldots, 4), \quad$ and $\quad w_{i}=1 / 36$ $(i=5, \ldots, 8)$. Mass and momentum are defined to be the first two moments of the distribution function respectively, and conservation of these quantities require

$$
\rho=\sum_{i} N_{i}=\sum_{i} N_{i}^{(e)},
$$

$$
\rho \mathbf{u}=\sum_{i} N_{i} \mathbf{c}_{i}=\sum_{i} N_{i}^{(e)} \mathbf{c}_{i}
$$

To apply the lattice Boltzmann equation to a range of flow problems, an internal or external force term may need to be added to Eq. (11), the exact form of which depends on the mechanics in question. A general representation of forcing terms within the LBE framework that considers discrete lattice effects has been proposed by Guo et al. [4]. With the intention of deriving Eqs. (8) and (9), a spatial and temporal microscopic term, $S_{i}(\mathbf{x}, t)$, is introduced into the D2Q9 lattice Boltzmann equation $[2,5]$ :

$$
N_{i}\left(\mathbf{x}+\mathbf{c}_{i}, t+1\right)=N_{i}(\mathbf{x}, t)+\omega\left[N_{i}^{(e)}(\mathbf{x}, t)-N_{i}(\mathbf{x}, t)\right]+S_{i}(\mathbf{x}, t),
$$

and we take this source term to be at least $O(\epsilon)$ :

$$
S_{i}=\epsilon S_{i}^{(1)}+\epsilon^{2} S_{i}^{(2)}+\cdots,
$$

that is, there is no equilibrium term, $S_{i}^{(0)}$.

The first- and second-order source terms that ensure that the axisymmetric Navier Stokes Eqs. (8) and (9) are recovered in the macroscopic limit are [1]

$$
\begin{gathered}
S_{i}^{(1)}=-\frac{w_{i} \rho u_{y}}{y}, \\
S_{i}^{(2)}=\frac{3 w_{i}}{y}\left[\frac{c_{i y}^{2}}{2}\left(\rho u_{x} \partial_{x} u_{y}-\frac{3 u_{y} \omega}{2} Q_{x x}-3 u_{y} \omega Q_{y y}-\frac{\rho u_{y}^{2}}{y}\right)\right. \\
-c_{i x}\left(\frac{6 \nu}{6 \nu+1} Q_{x y}+\frac{\rho}{6} \partial_{x} u_{y}-\rho u_{x} u_{y}\right)+c_{i y}(1-12 \nu) \\
\left.\times\left(\frac{1}{2(1+6 \nu)} Q_{y y}+\frac{\rho u_{y}}{y}-\rho u_{y}^{2}\right)\right],
\end{gathered}
$$

where

$$
Q_{\alpha \beta}=\sum_{i}\left(N_{i}-N_{i}^{(e)}\right) c_{i \alpha} c_{i \beta}
$$

We note that along the axis of symmetry, i.e., $y=0$, singular source terms of type $1 / y$ are evaluated using L'Hôpital's rule. After applying this formula, we are only left with terms

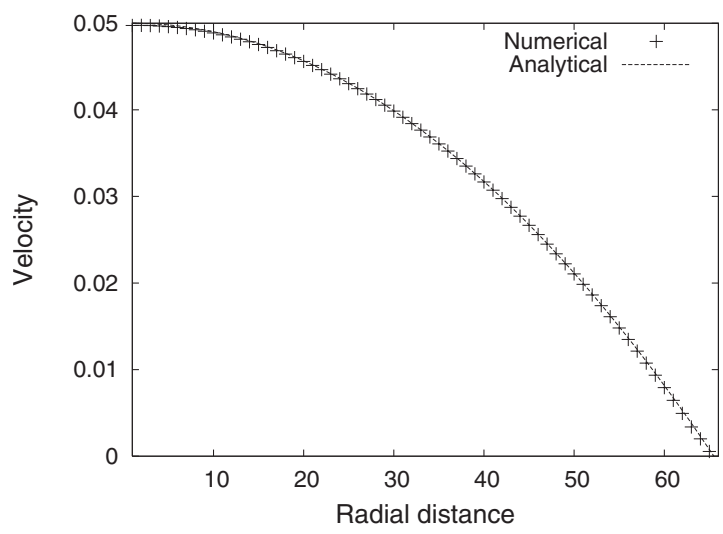

FIG. 1. Analytical and numerical solutions for Hagen-Poiseuille flow. 
TABLE I. Dependence of the error in the centerline velocity on lattice size.

\begin{tabular}{lc}
\hline \hline Lattice & Error in centerline velocity \\
\hline $128 \times 32$ & 0.00041 \\
$256 \times 64$ & 0.00010 \\
$512 \times 128$ & 0.00003 \\
$1024 \times 256$ & 0.00001 \\
\hline \hline
\end{tabular}

containing a factor $\partial_{y}(\cdot)$ or $u_{y}$. Since $u_{y}(0)=0$ and all resulting derivatives evaluated at $y=0$ are zero, we have $S_{i}(0)=0$ and therefore we only need to compute $S_{i}$ from $y=1$ to $y$ $=H$.

\section{NUMERICAL RESULTS}

\section{A. Hagen-Poiseuille flow}

We validate the model by simulating flows for which there are known exact solutions. The exact solution, $U(r)$, of the axisymmetric Navier-Stokes equations (3)-(5) to the steady, laminar flow of a viscous fluid through a pipe of radius $a$ is found to be

$$
U(r)=U_{0}\left(1-\frac{r^{2}}{a^{2}}\right),
$$

where

$$
U_{0}=\frac{G a^{2}}{4 \nu \rho}
$$

is the maximum velocity in the pipe and $G$ is the pressure gradient.

We construct a $256 \times 64$ D2Q9 lattice domain with a line of symmetry at $y=0$ and a solid wall at $y=64$. The no-slip boundary condition is applied at the wall using the interpolation scheme, as described in [6]. We impose an inflow and outflow condition as detailed in [7] with maximum velocity $U_{0}=0.05$.

Figure 1 plots the analytical solution (solid line) and the numerical predictions for Hagen-Poiseuille flow. The graph shows very good agreement between the analytical and numerical solutions, thus confirming the validity of the axisymmetric lattice Boltzmann equation proposed in [1].

To demonstrate mesh convergence we construct three additional meshes, one coarser and two finer than the one mentioned above. The coarse grid has dimensions $128 \times 32$ lattice units and the finer meshes have dimensions $512 \times 128$

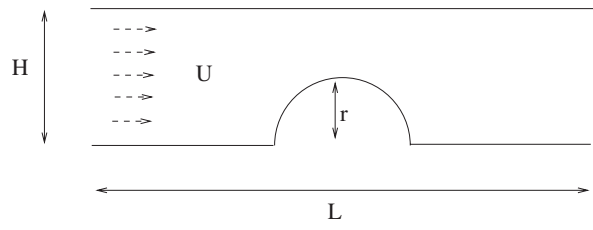

FIG. 2. Computational domain for axisymmetric flow over a sphere.
TABLE II. Comparison of drag coefficient for axisymmetric flow over a sphere using a second-order interpolation boundary

\begin{tabular}{|c|c|c|c|c|}
\hline $\mathrm{Re}$ & $D$ & $\bar{C}_{D}$ & $C_{D}=24 / \mathrm{Re}$ & Difference $(\%)$ \\
\hline 0.01 & 40 & 2402.1 & 2400 & 0.08 \\
\hline 0.01 & 60 & 2405.5 & 2400 & 0.2 \\
\hline 0.03 & 40 & 800.7 & 800 & 0.08 \\
\hline 0.03 & 60 & 801.6 & 800 & 0.2 \\
\hline 0.05 & 40 & 480.3 & 480 & 0.08 \\
\hline 0.05 & 60 & 481 & 480 & 0.2 \\
\hline 0.07 & 40 & 343.1 & 342.86 & 0.08 \\
\hline 0.07 & 60 & 343.5 & 342.86 & 0.2 \\
\hline 0.09 & 40 & 266.9 & 266.67 & 0.08 \\
\hline 0.09 & 60 & 267.2 & 266.67 & 0.2 \\
\hline 0.1 & 40 & 240.2 & 240 & 0.08 \\
\hline 0.1 & 60 & 240.5 & 240 & 0.2 \\
\hline 0.2 & 40 & 120.1 & 120 & 0.08 \\
\hline 0.2 & 60 & 120.2 & 120 & 0.2 \\
\hline 0.3 & 40 & 80.1 & 80 & 0.1 \\
\hline 0.3 & 60 & 80.2 & 80 & 0.3 \\
\hline 0.5 & 40 & 48.1 & 48 & 0.2 \\
\hline 0.5 & 60 & 48.2 & 48 & 0.4 \\
\hline 0.7 & 40 & 34.4 & 34.286 & 0.3 \\
\hline 0.7 & 60 & 34.46 & 34.286 & 0.5 \\
\hline 1 & 40 & 24.2 & 24 & 0.8 \\
\hline 1 & 60 & 24.2 & 24 & 0.8 \\
\hline 2 & 40 & 12.3 & 12 & 2.5 \\
\hline 2 & 60 & 12.3 & 12 & 2.5 \\
\hline 3 & 40 & 8.4 & 8 & 5 \\
\hline 3 & 60 & 8.4 & 8 & 5 \\
\hline
\end{tabular}
scheme.

and $1024 \times 256$. Note that the mesh refinement factor is 2 . Table I shows the error in the computed centerline velocity, $u(0)$. The decrease in the error as the mesh is refined demonstrates that the method is second order.

\section{B. Stokes' flow over a sphere}

Consider the flow of an incompressible, viscous fluid around a sphere. The Reynolds number for this problem is defined to be

$$
\operatorname{Re}=\frac{2 U R}{\nu}
$$

where $R$ is the radius of the sphere and $U$ is the free-stream velocity. If $\mathrm{Re}$ is small (typically $\mathrm{Re} \ll 1$ ) then Stokes' law is valid for the drag force, $F_{D}$, on the sphere in an infinite expanse of fluid [8]:

$$
F_{D}=6 \pi \mu R U
$$

where $\mu$ is the shear viscosity of the fluid. The drag coefficient, $C_{D}$, is defined as 


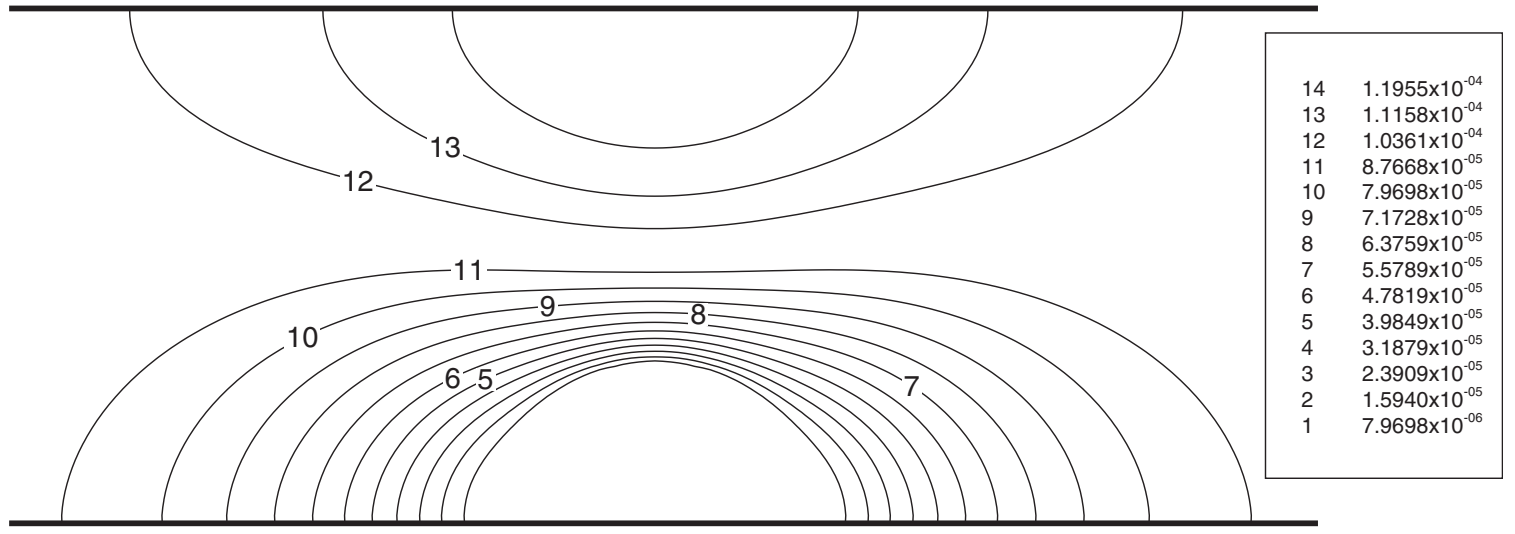

FIG. 3. Streamwise velocity contours for axisymmetric flow over a sphere at $\mathrm{Re}=0.01$.

$$
C_{D}=\frac{F_{D}}{0.5 \rho U^{2} A},
$$

where $A$ is the projected area of the obstacle in the plane perpendicular to the flow. For Stokes' flow over a sphere, $A=\pi R^{2}$, so the drag coefficient is

$$
C_{D}=\frac{24}{\operatorname{Re}} .
$$

We compute the drag coefficient using the axisymmetric LBM given by Eqs. (11), (17), and (18), and compare the results with the approximation given by Eq. (25).

We construct a $L \times H$ D2Q9 lattice domain with a line of symmetry at $y=0$ and place a sphere of radius $R$ at $(x=L / 2, y=0)$, as shown in Fig. 2. The second-order interpolation scheme, as described in the article by Yu et al. [6], is used to apply the boundary conditions on the cylinder. As Eq. (25) is valid in an infinite domain we apply symmetry conditions at the north and south boundaries and specify a uniform velocity, $U$, at the inlet.

Table II shows the computed drag coefficient $\left(\bar{C}_{D}\right)$, the Stokes approximation for the drag and the relative difference between the two for different Reynolds numbers. To determine whether or not different flow parameters affect the computation we calculated the drag coefficient twice for each
Reynolds number, changing the radius of the sphere and the fluid viscosity while keeping the velocity $U=\operatorname{Re} / 100$. In all simulations we kept $L / H=5$ and $R / H=0.3$. Excellent agreement is obtained with the difference between the analytical formula for the drag coefficient and the lattice Boltzmann predictions below $0.2 \%$ for $\operatorname{Re}<0.5$. Figures 3 and 4 plot the streamwise and normal velocity contours, respectively, for the axisymmetric flow over a sphere at $\operatorname{Re}=0.01$. The contour heights are displayed in these figures. The plots show the expected characteristics associated with this flow, including almost symmetry of the velocity field fore and aft of the sphere, decaying axial velocity approaching the sphere, and a positive radial velocity in front of the sphere and a negative one behind. Figures 5 and 6 plot the streamwise and normal velocity contours, respectively, for the axisymmetric flow over a sphere at $\operatorname{Re}=100$. These show the development of a boundary layer on the upstream portion of the sphere and a large toroidal vortex in the near wake.

From Table II we see that as the Reynolds number increases beyond 0.5 the relative difference between the approximations gradually increases. This is to be expected, since Stokes' formula (25) is only valid when the inertia terms in the Navier-Stokes equations can be neglected, i.e., when the Reynolds number is small. When Re is large the following empirical formula for the drag coefficient on a sphere has been suggested [9]:
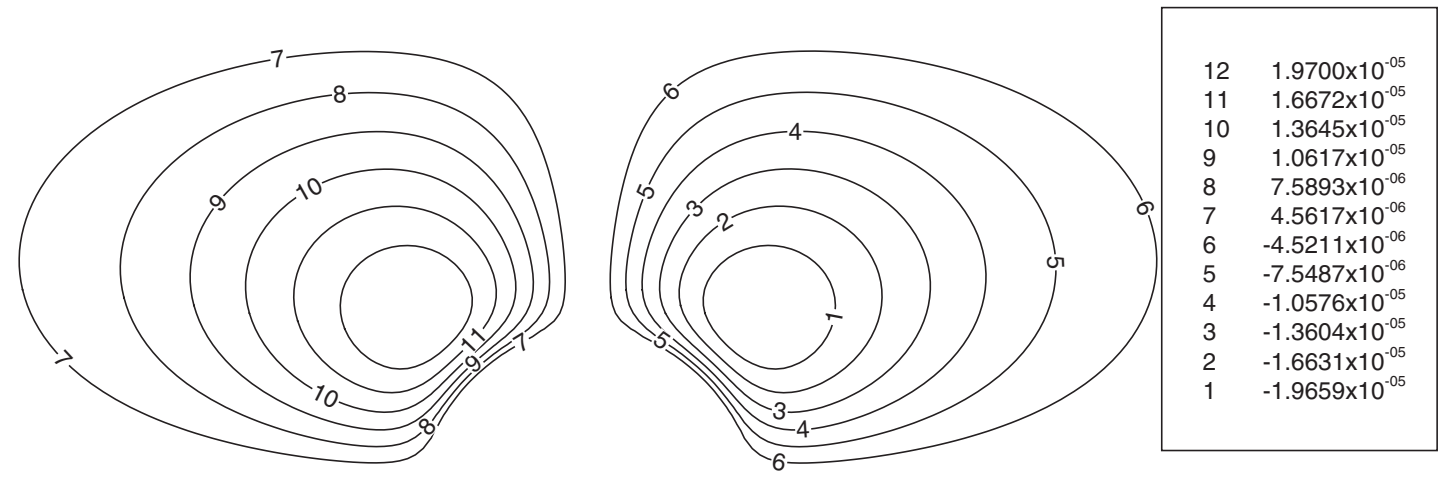

FIG. 4. Normal velocity contours for axisymmetric flow over a sphere at $\mathrm{Re}=0.01$. 


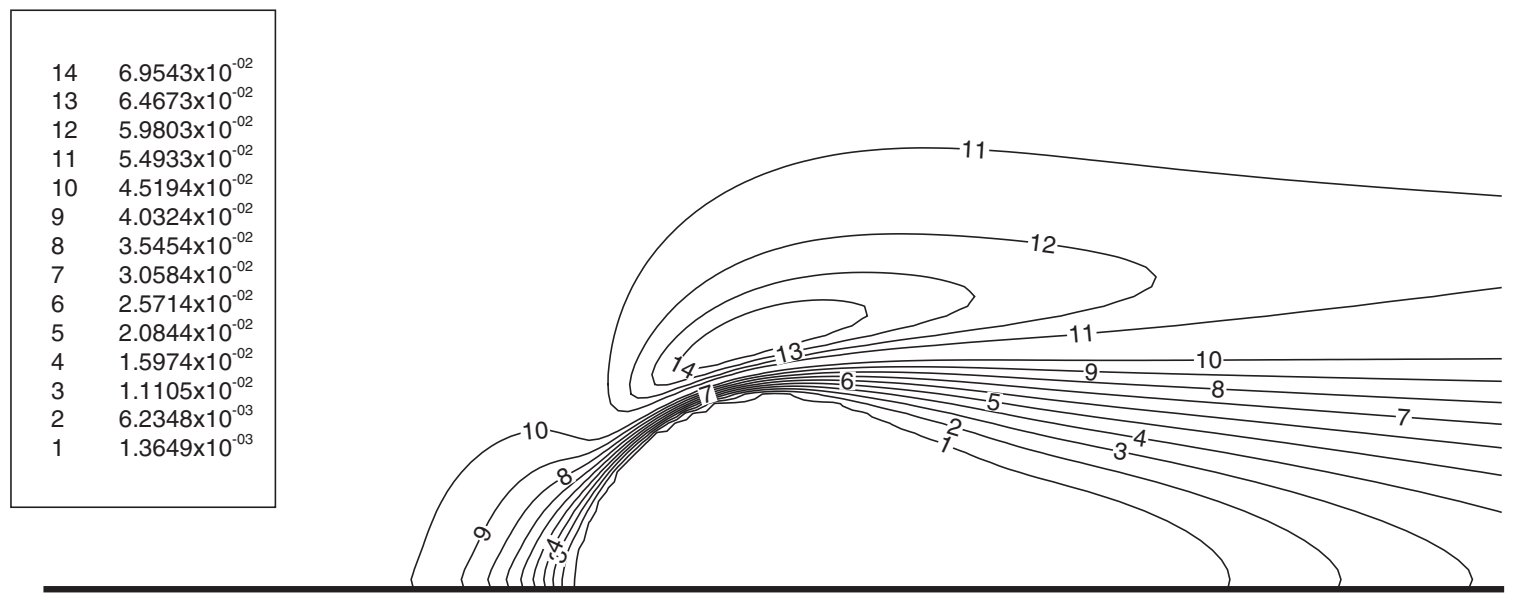

FIG. 5. Streamwise velocity contours for axisymmetric flow over a sphere at $\mathrm{Re}=100$.

$$
C_{D}=\left(\sqrt{\frac{24}{\operatorname{Re}}}+0.5407\right)^{2}
$$

Table III shows the drag coefficient found by Eq. (26) and the numerical approximations for larger Reynolds numbers. In all cases $U=0.05$ and $D=40$. We clearly see that the lattice Boltzmann predictions approach the value given by the empirical formula as Re is increased. Figure 7 plots the drag coefficient as a function of Reynolds number and clearly shows the excellent agreement between the analytic formula for $C_{D}$ in axisymmetric Stokes' flow and the lattice Boltzmann predictions. The graph also highlights the departure of the numerical approximation of the drag coefficient from Stokes' formula at $\operatorname{Re} \approx 1$ and its subsequent approach to the empirical formula (26) as Re is increased further.

\section{CONCLUSIONS}

In this paper we have evaluated a modified axisymmetric lattice Boltzmann equation which has been shown to be a second-order approximation to the axisymmetric NavierStokes equations [1]. Unlike previous models, this one correctly applies the Chapman-Enskog analysis to the LBE and fully exposes the coupling between the two additional source terms through the nonequilibrium contribution to the D2Q9 distribution function. Therefore, it recovers the correct spatial derivatives in the macroscopic limit. These source terms are geometric forces which are consistent with the general form for additional LBE forces as found by Guo et al. [4]. Moreover, these source terms are more local than those derived by Halliday et al. [2] since they are given mainly in terms of moments of the nonequilibrium distribution function. This has the practical advantage of reducing the amount of numerical differentiation which is not only more sympathetic to the general lattice Boltzmann philosophy, but is also likely to improve computational efficiency and stability. This last point is as yet unproven but a local stability analysis could be conducted using a Fourier-space formulation (as described in Ref. [10]). We first validated the model by simulating Hagen-Poiseuille flow. This flow has a well-known analytical solution, with which our numerical predictions are

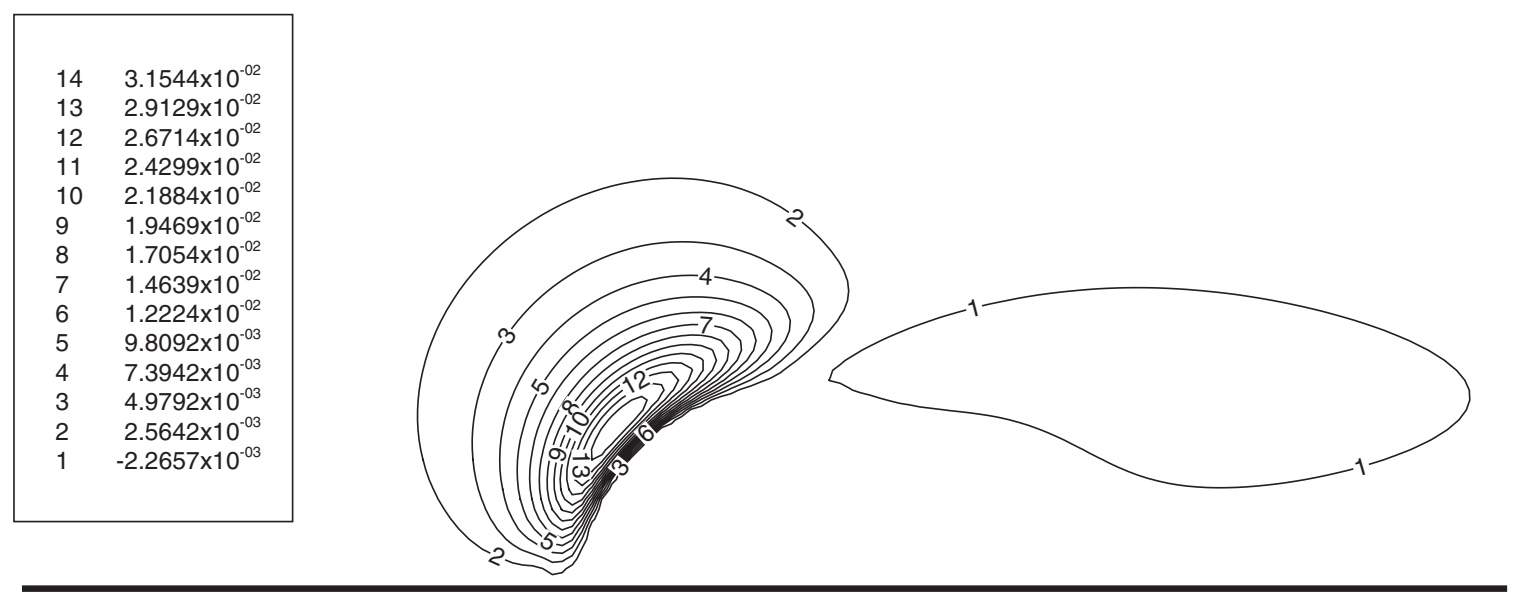

FIG. 6. Normal velocity contours for axisymmetric flow over a sphere at $\mathrm{Re}=100$. 
TABLE III. Comparison of drag coefficient for axisymmetric flow over a sphere for larger Reynolds numbers.

\begin{tabular}{lccc}
\hline \hline $\operatorname{Re}$ & $\bar{C}_{D}$ & $C_{D}$ & Difference $(\%)$ \\
\hline 10 & 3.2101 & 4.3677 & 26.50 \\
20 & 2.1240 & 2.6760 & 20.63 \\
40 & 1.5195 & 1.7300 & 12.17 \\
100 & 1.0726 & 1.0621 & 0.99 \\
150 & 0.8761 & 0.8849 & 0.99 \\
\hline \hline
\end{tabular}

shown to agree very well. We then applied our equation to Stokes' flow over a sphere and found excellent agreement between the analytical and numerical approximations for the drag coefficient for all $\operatorname{Re}<1$ (i.e., when Stokes' law is valid). At higher Reynolds numbers our results agree with an empirical formula for the drag based on experimental evidence.

This modified axisymmetric LBE can also be easily combined with existing Rothmann-Keller-type multiphase lattice Boltzmann models [11]. From a micro or mesoscopic view point the segregation of two fluids is due to interparticle forces. LBE methods are therefore in a strong position compared to traditional macroscopic techniques based on continuum mechanics since these particle interactions can be incorporated into the evolution of the distribution function. As a result, a multiphase LBE model should not track interfaces but rather let them emerge spontaneously from the underlying dynamics.

Although LBE methods for fluid-fluid multiphase flows are able to reproduce basic interfacial phenomena such as spinoidal decomposition in binary fluids, the RayleighTaylor instability, etc. [12], many existing methods do not fully utilize the origins of the method in kinetic theory. A review of existing LBE methods for fluid-fluid multiphase and their capabilities and limitations may be found in Nourgaliev et al. [12].

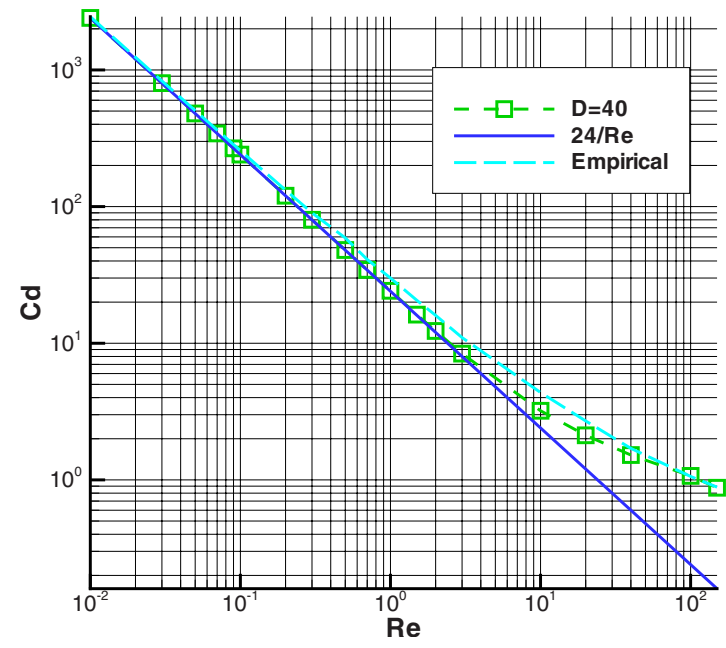

FIG. 7. (Color online) Graph showing $C_{D}$ as a function of Re for axisymmetric flow over a sphere.

Gunstensen et al. [13] proposed a LBM for multiphase flows that combined the single-phase LBM of McNamara and Zanetti [14] with the multiphase lattice gas algorithm of Keller and Rothman [15]. An important contribution in this LBE model is the introduction of a perturbation step in order to recover Laplace's formula at an interface in which a binary fluid collision operator is added to the post collision state at sites near the interface. Reis and Phillips [16] proposed a modification of the model of Gunstensen et al. [13] for binary fluids in which the two-phase operator is adjusted and shown to recover the single phase Navier-Stokes equations, with an appropriate source term to model surface tension, in the macroscopic limit. Extensive numerical experiments for planar flows showed that the model was capable of predicting flows with large density ratios.
[1] T. Reis and T. N. Phillips, Phys. Rev. E 75, 056703 (2007).

[2] I. Halliday, L. A. Hammond, C. M. Care, K. Good, and A. Stevens, Phys. Rev. E 64, 011208 (2001).

[3] Y. Qian, D. d'Humières, and P. Lallemand, Europhys. Lett. 17, 479 (1992).

[4] Z. Guo, C. Zheng, and B. Shi, Phys. Rev. E 65, 046308 (2002).

[5] K. N. Premnath and J. Abraham, Phys. Rev. E 71, 056706 (2005).

[6] D. Yu, R. Mei, and W. Shyy, AIAA J. 2003-953, 2003 (2003).

[7] D. Yu, R. Mei, L. Luo, and W. Shyy, Prog. Aerosp. Sci. 39, 329 (2003).

[8] H. Lamb, Hydrodynamics (Dover Publications, New York,
1945).

[9] R. Bird, W. Stewart, and E. Lightfoot, Transport Phenomena (Wiley, New York, 2002).

[10] P. Lallemand and L. S. Luo, Phys. Rev. E 61, 6546 (2000).

[11] T. Reis, Ph.D. thesis, Cardiff University, 2007.

[12] R. R. Nourgaliev, T. G. Theofanous, and D. D. Joseph, Int. J. Multiphase Flow 29, 117 (2003).

[13] A. K. Gunstensen, D. H. Rothman, S. Zaleski, and G. Zanetti Phys. Rev. A 43, 4320 (1991).

[14] G. R. McNamara and G. Zanetti, Phys. Rev. Lett. 61, 2332 (1988).

[15] D. Rothman and J. Keller, J. Stat. Phys. 52, 1119 (1988).

[16] T. Reis and T. N. Phillips, J. Phys. A 40, 4033 (2007). 